\title{
Critical Examination of a Transformation to Vector-Mean Coordinates
}

\author{
J. C. Kaimal and C. N. Touart
}

Air Force Cambridge Research Laboratories, Bedford, Mass.

22 August 1966 and 4 January 1967

\section{Introduction}

For more than a decade various investigators (Cramer et al., 1957; Haugen, 1959; Cramer et al., 1961; Miyake, 1965) have used the following transformation to generate Cartesian wind components from data sensed in spherical coordinates, as with a bivane or a hot-wire system. Using symbols $u^{0}, v^{0}$ and $w^{0}$ to denote velocity components,

$$
\begin{aligned}
u^{0} & =|\mathbf{V}| \cos \left(E-E^{*}\right) \cos \left(A-A^{*}\right), \\
v^{0} & =|\mathbf{V}| \cos \left(E-E^{*}\right) \sin \left(A-A^{*}\right), \\
w^{0} & =|\mathbf{V}| \sin \left(E-E^{*}\right)
\end{aligned}
$$

where

$$
\begin{aligned}
& A^{*}=\tan ^{-1}\left(\frac{\overline{|\mathbf{V}| \sin E}}{\overline{|\mathbf{V}| \cos E}}\right], \\
& E^{*}=\tan ^{-1}\left[\frac{\overline{|\mathbf{V}| \cos \left(E-E^{*}\right) \sin A}}{\overline{|\mathbf{V}| \cos \left(E-E^{*}\right) \cos A}}\right] .
\end{aligned}
$$

Here $\mathrm{V}, A$ and $E$ represent the instantaneous wind vector and its measured azimuth and elevation angles, respectively.

For convenience we shall refer to this as the "Great Plains transformation" after one of its earliest uses. It 
is clearly the conventional conversion to Cartesian components, but with angles modified by subtracting $A^{*}$ and $E^{*}$.

Users of the transformation have generally held that the components thus generated are those appropriate to a vector-mean coordinate system, i.e., the system in which the $u$-axis lies along the vector mean of the sample and the $v$-and $w$-axes are normal to it. This is a very plausible contention since $\overrightarrow{v^{0}}=\overrightarrow{w^{0}}=0$ for any sample, as can be readily demonstrated. However, we find that exactly what the transformation does, and how well it converts to the desired Cartesian coordinates, depend crucially on the cause and magnitude of $E^{*}$. The purpose of this note is to point out the ambiguities and possible sources of error in the transformation.

\section{Significance of $E^{*}$}

In general $E^{*}$ has no physical significance although occasionally it has been assumed to be the inclination angle of the vector-mean wind. In practice, $E^{*}$ is usually comparable in magnitude to $\bar{E}$, the scalar mean of the elevation angle. The maximum value of $E^{*}$ in the Prairie Grass data (Haugen, 1959) is $28.6^{\circ}$ and values between $10^{\circ}$ and $20^{\circ}$ are common.

A non-zero value of $E^{*}$ can be expected to arise from one of two causes:

1) If $\overline{\mathbf{V}}$, the vector mean, is actually inclined to the horizontal for any sample;

2) If there is an intentional or accidental offset of the inclination angle in the measurement system.

If the vector-mean wind is inclined to the horizontal, due to choice of terrain or record length, and the nonzero $E^{*}$ is due exclusively to it, then the Great Plains transformation yields only approximations to velocity components in vector-mean space, and the degree of approximation deteriorates with increasing magnitude of $E^{*}$. The principal reason for this is that spherical coordinates do not transform between tilted coordinate systems through a simple subtraction of angles. This case is treated in more detail in the following section.

If the vector-mean wind lies in the horizontal plane, any non-zero $E^{*}$ will be due exclusively to offsets in the measurement. Then the transformation does convert the measurements accurately into Cartesian coordinates with the $u$-axis along the vector-mean direction which, in this case, is horizontal by definition. However, if $E^{*}$ results from a combination of instrument offset and inclination in the vector mean the problem becomes more complex.

The discussion to follow is an analysis of the first case where $E$ and $A$ represent the true elevation and azimuth angles measured by a correctly oriented instrument with no built-in offsets. Errors involved in considering the derived quantities to be vector-mean components are then estimated.

\section{Analysis of the Great Plains transformation}

For comparison we present below a transformation which converts correctly to vector-mean coordinates. In this correct transformation the $u$-axis is oriented along the vector-mean direction, the $v$-axis is the normal to it in the horizontal plane and the $v$-axis is normal to both the $u$-and $v$-axes so as to form a right-handed coordinate system. Using $\widetilde{A}$ and $\widetilde{E}$ to denote the azimuth and elevation angles of the vector-mean direction, we have

$$
\begin{aligned}
u & =|\mathbf{V}|[\cos E \cos (A-\tilde{A}) \cos \tilde{E}+\sin E \sin \tilde{E}], \\
v & =|\mathbf{V}|[\cos E \sin (A-\widetilde{A})], \\
w & =|\mathbf{V}|[-\cos E \cos (A-\tilde{A}) \sin \tilde{E}+\sin E \cos \tilde{E}],
\end{aligned}
$$

where

$$
\begin{aligned}
& \tilde{A}=\tan ^{-1}\left(\overline{\frac{|\mathbf{V}| \cos E \sin A}{\sqrt{\mathbf{V} \mid \cos E \cos A}}}\right),
\end{aligned}
$$

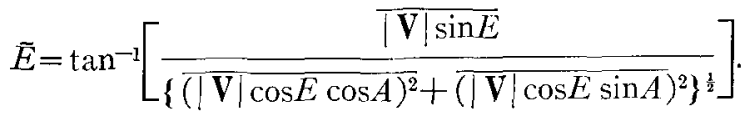

The error introduced in $u^{0}, v^{0}$ and $w^{0}$ are evaluated in two steps. That resulting from incorrectness of the form in Eq. (1) is examined first, assuming $A^{*}$ and $E^{*}$ to be the correct azimuth and elevation angles of $\mathrm{V}$, i.e., $A^{*}=\widetilde{A}$ and $E^{*}=\widetilde{E}$. The discrepancy arising from $A^{*}$ and $E^{*}$ not representing the correct angles is then dealt with separately. For convenience the former will be referred to as the "transformation error," the latter as the "angle error," and the sum of both as the "total error."

a. Analysis of the transformation error. Assuming $A^{*}=\widetilde{A}$ and $E^{*}=\widetilde{E}$, and denoting the velecity components thus obtained by $\hat{u}, \hat{v}$ and $\hat{w}$, Eq. (1) becomes

$$
\begin{aligned}
& \hat{u}=|\mathbf{V}| \cos (E-\tilde{E}) \cos (A-\widetilde{A}), \\
& \hat{v}=|\mathbf{V}| \cos (E-\widetilde{E}) \sin (A-\widetilde{A}), \\
& \hat{v}=|\mathbf{V}| \sin (E-\widetilde{E}) .
\end{aligned}
$$

Since $\widetilde{A}$ and $\widetilde{E}$ have definite physical significance, this transformation is easier to visualize geometrically than the one in Eq. (1). Examining the expression for $\hat{w}$, which is the simplest of three, one finds that it actually defines the length of the perpendicular dropped from the instantaneous vector $\mathbf{V}$ to the surface generated by rotating $\overline{\mathbf{V}}$ about the true vertical (see Fig. 1). The surface of rotation is a cone of constant $\bar{E}$ and consequently the angle between any instantaneous vector $V$ and the cone will always be $(E-\tilde{E})$. However, the orientation of the perpendicular, i.e., $|V| \sin (E-\bar{E})$, will be different for each different vector $V$. Therefore, $\hat{v}$ does not represent a velocity component along any fixed direction. 


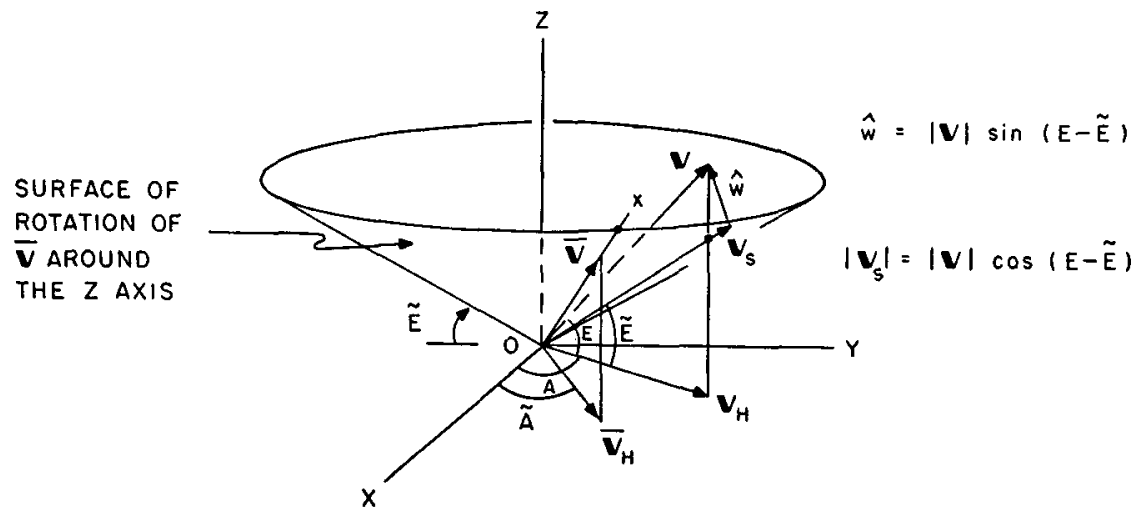

FIG. 1. Geometrical construction of $\hat{w}$. Axes $X, Y, Z$ are the reference axes of the instrument ( $Z$-axis considered vertical) and $x$ is the $u$-axis along the vector-mean direction.

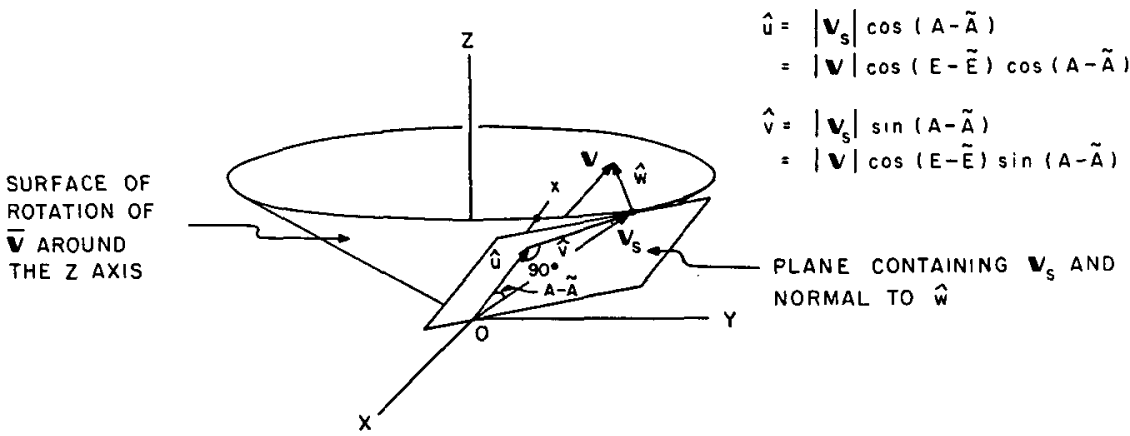

Fig. 2. Geometrical construction of $\hat{u}$ and $\hat{v}$. Axes $X, Y$, and $Z$ and $x$ have the same meaning as in Fig. 1 .

With similar constructions for $\hat{u}$ and $\hat{v}$ it is shown (see Fig. 2) that they too differ from the correct $u$ and $v$ components. Both $\hat{u}$ and $\hat{v}$ lie in a plane tangent to the cone surface. They will be oriented so as to form a right-angled triangle with $\mathbf{V}_{s}$, the projection of $\mathbf{V}$ on the cone surface, as the hypotenuse and an angle equal to $(A-\widetilde{A})$ at the origin. Their orientation being different for each different vector $V$, they also do not represent velocity components along fixed directions.

The discrepancy in each component can be evaluated by simply subtracting (3) from (5). These can be expressed as

$$
\begin{aligned}
& \frac{\hat{u}-u}{|\mathbf{V}|}=-\sin E \sin \tilde{E}[1-\cos (A-\tilde{A})], \\
& \frac{\hat{v}-v}{|\mathbf{V}|}=\sin (A-\tilde{A})[\cos (E-\tilde{E})-\cos E], \\
& \frac{\hat{w}-w}{|\mathbf{V}|}=-\cos E \sin \tilde{E}[1-\cos (A-\tilde{A})] .
\end{aligned}
$$

The difference between the two transformations vanishes only when $\widetilde{E}=0$ or $(A-\widetilde{A})=0$.

$b$. Analysis of the angle error. It can be shown from Eqs. (2b) and (4b) that

$$
\frac{\tan E^{*}}{\tan \bar{E}}=\frac{\left|\overline{\mathbf{V}}_{H}\right|}{\left|\overline{\mathbf{V}}_{H}\right|},
$$

where $\mathrm{V}_{I I}$ is the horizontal projection of $\mathrm{V}$. The right side of the equation is the ratio of the magnitude of the vector mean of $\mathbf{V}_{H}$ to its scalar mean. By assuming a circular normal distribution for $\mathbf{V}_{H}$ and a maximum value of $30 \%$ for $\sigma(u) /\left|\overline{\mathbf{V}}_{H}\right|$, the above ratio is estimated (Brooks et al., 1946) to be 0.97. Using this estimate, the angle error in the $w$-component can be expressed as

$$
\frac{w^{0}-\imath \hat{w}}{|\mathbf{V}|} \approx 0.015 \cos (E-\bar{E}) \sin 2 \widetilde{E}
$$

Next, assuming no systematic correlation between the instantaneous elevation angle and the horizontal ve- 

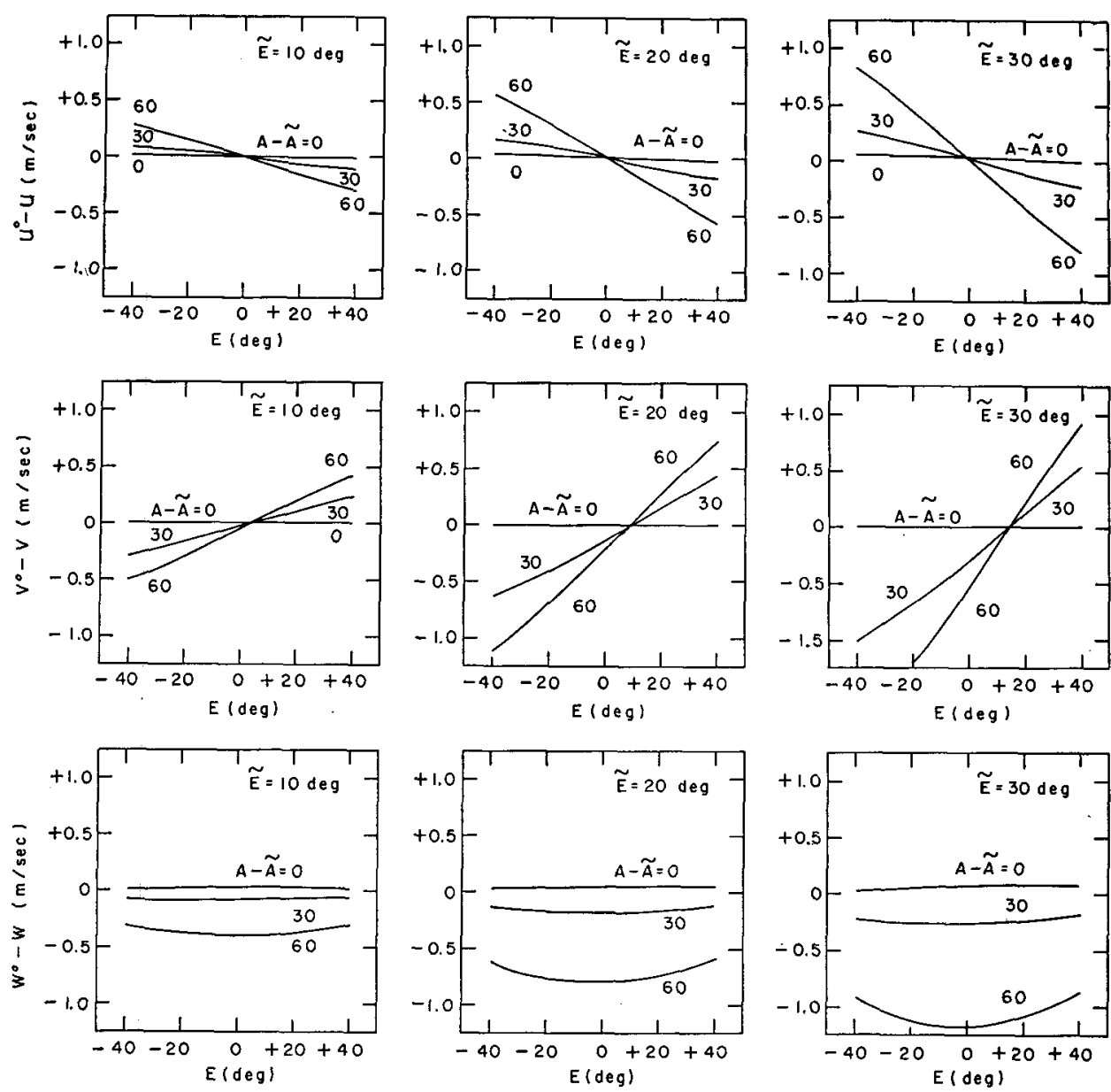

Fig. 3. Departures in computed $u^{0}, v^{0}$, and $w^{0}$ from the correct vector-mean components plotted as a function of angular fluctuations. Estimates shown are for a typical wind speed of $5 \mathrm{~m} \mathrm{sec}-1$.

locity components, the following expressions can be derived for errors in the $u$-and $v$-components:

$$
\begin{aligned}
& \frac{u^{0}-\hat{u}}{|\mathbf{V}|} \approx-0.015 \sin (E-\bar{E}) \sin 2 \tilde{E} \cos (A-\widetilde{A}), \\
& \frac{v^{0}-\hat{v}}{|\mathbf{V}|} \approx-0.015 \sin (E-\tilde{E}) \sin 2 \tilde{E} \sin (A-\widetilde{A}) .
\end{aligned}
$$

Combining the "transformation" and the "angle" errors one can get estimates of the "total" error. These are plotted in Fig. 3 for a typical wind speed of $5 \mathrm{~m} \mathrm{sec}^{-1}$ and three different values of $\widetilde{E}$. In all three wind components the error increases steadily with $\vec{E}$. The error appears to increase also with azimuth fluctuations $(A-\widetilde{A})$ as long as $\widetilde{E} \neq 0$. The largest error occurs in the w-component where it is comparable in magnitude to typical values of the component itself.

\section{Conclusions}

Velocity components derived from the Great Plains transformation do not represent vector-mean compo- nents except when the vector-mean direction is horizontal. Deviations from true vector-mean components increase with inclination of the vector-mean wind.

If vector-mean components are required, the transformation to be used is Eq. (3); here it is imperative that all instrument offsets in the elevation angle be determined as accurately as possible and removed prior to transformation. If, on the other hand, the desired components are to be in the "natural" coordinate system (where the $u$-axis is along the horizontal projection of the vector-mean and w-axis is vertical), which is perhaps more relevant for energy flux studies, Eq. (1) may be used provided the vector-mean direction is known to be horizontal. In this case $E^{*}$ accurately represents the offset in the measurement system. If there is any uncertainty about the orientation of the vector-mean, the correct procedure for transforming to natural coordinates is to remove all offsets in the elevation angle before the transformation and to use $\mathrm{Eq}$. (1) but with $E^{*}$ deleted.

Acknowledgments. The authors wish to express their appreciation to Dr. M. L. Barad and Dr. D. A. Haugen 
for their encouragement and help in preparing this paper and for their critical review of the manuscript.

\section{REFERENCES}

Brooks, C. E. P., C. S. Durst and N. Carruthers, 1946: Upper winds over the world. Part I: The frequency distribution of winds at a point in the free air. Quart. J. Roy. Meteor. Soc., 72 , p 55.

Cramer, H. E., G. C. Gill and F. A. Record, 1957 : Heated thermocouple anemometers and light bivanes-Massachusetts Institute of Technology. Exploring the Atmosphere's First Mile, Vol. I, New York, Pergamon Press, p. 241.
F. A. Record, J. E. Tillman and H. C. Vaughan, 1961: Studies of the spectra of the vertical fluxes of momentum, heat and moisture in the atmospheric boundary layer. Annual Report, Dept. Meteor., Massachusetts Institute of Technology, 130 pp.

Haugen, D. A., 1959: Reduction and spectral analyses of M.I.T. bivane data. Project Prairie Grass, A Field Program in Diffusion, Vol. 3, AFCRC-TR-58-235(III), Air Force Cambridge Research Center, 673 pp.

Miyake, M., $1965:$ A constant temperature wind component meter development and application. Final Report, Dept. of Atmospheric Sciences, University of Washington, $115 \mathrm{pp}$. 